\title{
AIRBORNE POLARIMETRIC, TWO-COLOR LASER ALTIMETER MEASUREMENTS OF LAKE ICE COVER: A PATHFINDER FOR NASA'S ICESAT-2 SPACEFLIGHT MISSION
}

\author{
David Harding ${ }^{\prime}$, Philip Dabney ${ }^{\prime}$, Susan Valett', Anthony Yu', Aleksey Vasilyev ${ }^{2}$ and April Kelly ${ }^{3}$ \\ ${ }^{1}$ NASA Goddard Space Flight Center, Greenbelt, MD 20771 \\ ${ }^{2}$ Sigma Space Corporation, Lanham, MD, 20706 \\ ${ }^{3}$ National Space Club Scholars Intern, Goddard Space Flight Center, Greenbelt, MD 20771
}

\begin{abstract}
The ICESat-2 mission will continue NASA's spaceflight laser altimeter measurements of ice sheets, sea ice and vegetation using a new measurement approach: micropulse, single photon ranging at $532 \mathrm{~nm}$. Differential penetration of green laser energy into snow, ice and water could introduce errors in sea ice freeboard determination used for estimation of ice thickness. Laser pulse scattering from these surface types, and resulting range biasing due to pulse broadening, is assessed using SIMPL airborne data acquired over icecovered Lake Erie. SIMPL acquires polarimetric lidar measurements at 1064 and $532 \mathrm{~nm}$ using the micropulse, single photon ranging measurement approach.
\end{abstract}

Index Terms - Lidar, sea ice, polarimetry, SIMPL, ICESat

\section{INTRODUCTION}

NASA's Ice Cloud and land Elevation Satellite (ICESat) acquired measurements of ice sheet elevations and their change, sea ice thickness, land topography and vegetation height from 2003 to 2009 [1-4]. ICESat used a traditional laser altimeter measurement approach, single-beam, singlepulse, analog waveform recording in the near infrared. The follow-on ICESat-2, scheduled for launch mid-decade, will use new approach, multi-beam, micropulse, photon counting in the green [5,6]. Differential green wavelength penetration into snow, ice and water poses a potential source of bias in the measurement of sea ice freeboard used to estimate ice thickness. Freeboard is the height of the sea ice surface relative to that of adjacent water in leads crossing through the ice. The airborne Slope Imaging Multipolarization Photon-counting Lidar (SIMPL) is being used to assess potential sources of error in freeboard determination in preparation for the ICESat-2 mission.

\section{METHODOLOGY}

SIMPL is a multi-beam laser altimeter developed through the NASA Earth Science Technology Office Instrument
Incubator Program [7]. SIMPL is a pathfinder for more efficient, next-generation spaceflight laser altimeters including ICESat-2. It simultaneously measures surface topography, roughness and slope as well as laser pulse scattering properties to differentiate surface types. The SIMPL transmitter is a short-pulse, $1064 \mathrm{~nm}$ (near infrared), plane-polarized micropulse laser that is frequency doubled to $532 \mathrm{~nm}$ (green) and split into four parallel, push-broom beams. The receiver employs single-photon detection with high timing precision at both wavelengths to determine the range to a target. The short pulse width and high timing precision achieves an $8 \mathrm{~cm}$ range precision per single detected photon. Upon aggregation of signal photons into a range histogram a measurement with a few $\mathrm{cm}$ resolution of pulse width is achieved. The impulse response from a flat, non-transparent surface has a full-width half-max pulse width of $\sim 15 \mathrm{~cm}$.

Received pulse broadening is due to surface roughness as well as light transmission into the target and resulting volume scattering. Due to limited near infrared (NIR) penetration into snow, ice and water the $1064 \mathrm{~nm}$ pulse broadening is primarily a measure of surface roughness whereas penetration in the green introduces additional broadening and a resulting range delay. SIMPL acquires reflected energy parallel (\|) and perpendicular $\left(\perp_{-}\right)$to the transmit pulse polarization plane, enabling a depolarization measurement at the two wavelengths that provides information on surface and volume scattering properties used in differentiating target types. Increasing scattering causes an increasing amount of 1 reflected energy, and an increase in the depolarization ratio $\mid / \|$. The depolarization signal is particularly useful in differentiating water and smooth ice, whose surface is specular, from rougher snow and ice. The signal from specular surfaces, consisting of single scattering reflections, contains only || energy. ICESat-2 will have high precision range resolution similar to SIMPL but will operate only at $532 \mathrm{~nm}$ with no polarization measurement. SIMPL's combination of highly resolved pulse broadening at two wavelengths and polarimetric differentiation of surface types provides a 

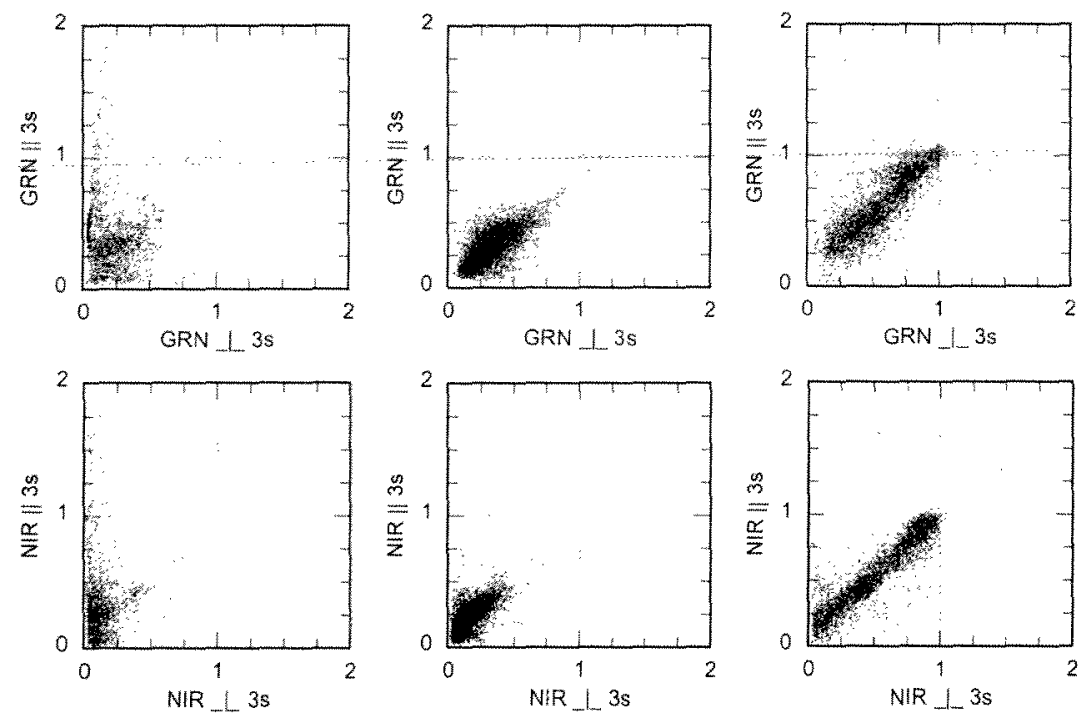

Figure 1. Green (top) and near infrared (bottom) parallel versus perpendicular received energy, normalized with respect to a snow reference target, for open water (left), translucent grey ice (middle) and opaque white ice (right). Results are for one of the four SIMPL beams.

unique set of measurements ideally suited to address biases in sea ice freeboard determination that might impact the ICESat-2 mission.

\section{INSTRUMENTATION}

Instrumentation details are provided in [7]. In summary, the SIMPL instrument consists of a multi-beam laser transmitter and receiver mounted on opposite sides of a thermally stable optical bench and sharing a common $20 \mathrm{~cm}$ aperture primary mirror. The laser transmitter is an $11 \mathrm{kHz}, 1064 \mathrm{~nm}$ microchip laser, frequency doubled to $532 \mathrm{~nm}$, with a fullwidth half-max pulse width of $1 \mathrm{nsec}(0.15 \mathrm{~m})$. The two colors are split into four plane-polarized beams and coalignment of the two colors is preserved. This co-alignment is a key feature, ensuring that the pulse-width measurements are acquired from the same target location. The 16 channel receiver splits the received energy for each beam into the two colors and each color is split into energy parallel and perpendicular to the transmit polarization plane, thereby providing the measure of backscatter depolarization. The solar background count rate is controlled by spatial filtering using a pinhole array and by spectral filtering using temperature-controlled narrow bandwidth filters. The receiver is fiber coupled to 16 Single Photon Counting Modules (SPCMs). To avoid range biases due to the long dead time of these detectors the probability of detection per laser fire on each channel is controlled to be below $30 \%$, using mechanical irises and flight altitude. The laser fire time and photon detection events are tagged with $0.1 \mathrm{nsec}$ precision, yielding a single-photon range precision of $\sim$ At typical aircraft flight speeds, each of the 16 channels acquires a single photon range every 5 to $15 \mathrm{~cm}$ along the four profiles providing a highly sampled measurements of surface properties. The nominal flight altitude was $4 \mathrm{~km}$ yielding $8 \mathrm{~m}$ spacing between the four beam profiles, providing a measure of surface slope at $24 \mathrm{~m}$ length scales.

\section{RESULTS FOR ICE COVERED LAKE ERIE}

SIMPL was flown aboard NASA's Lear-25 based at the Glenn Research Center, acquiring data over ice-covered Lake Erie in February, 2009. The ice cover, analogous to young sea ice formation, consisted of four types that, from youngest to oldest, were fresh skim ice, dark nilas ice, translucent new grey ice and more opaque new grey-white ice. The lake ice is covered by snow in some places and broken by open water leads and irregularly shaped polynya. Surface types were identified in nadir in-flight video and correlated with the SIMPL data using GPS time-tagging. For each of the 16 channels the number of signal photons over 0.1 second segments $(\sim 12 \mathrm{~m})$ were summed and the amplitudes normalized by a snow reference surface. The relative amplitudes of the parallel and perpendicular channels at the two wavelengths provide diagnostic signatures that aid in differentiating the surface types (e.g., Figure 1).

Accumulation of signal photon ranges acquired over $0.5 \mathrm{sec}$ $(\sim 60 \mathrm{~m})$ yields range histograms documenting pulse broadening for the four SIMPL channels. For an opaque new grey-white ice target green light pulse broadening due to penetration into the ice is only a few $\mathrm{cm}$ greater than that for NIR light, indicating the $532 \mathrm{~nm}$ range bias is very small (Figure 2, top). Perpendicular-polarization received signal at both wavelengths is present due to surface and volume multiple-scattering. For an open water specular surface (Figure 2, middle) NIR __ reflected energy is minimal as 

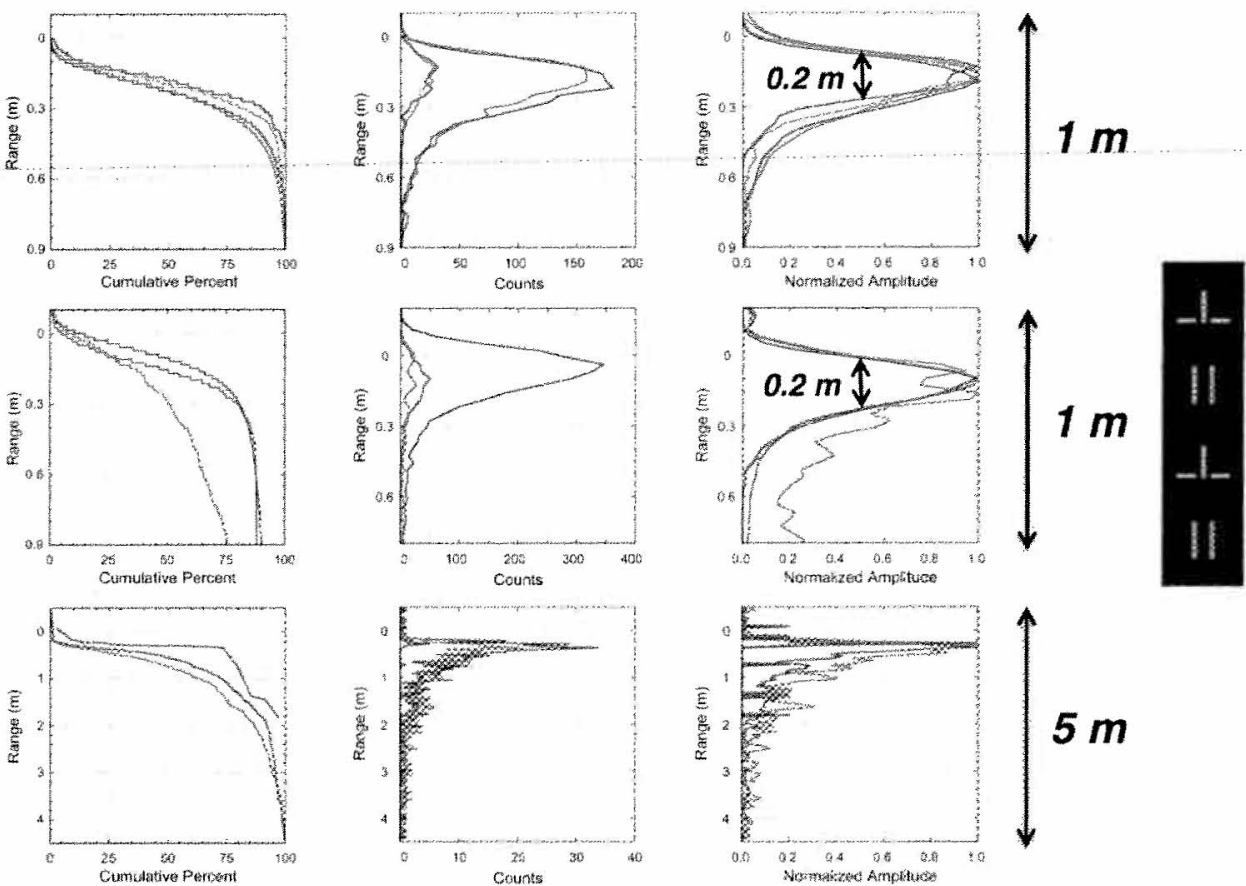

Figure 2. Range distributions for opaque new grey-white ice (top), water with a wind-roughened surface (middle) and water with a thin cover of skim ice (bottom) showing cumulative signal amplitude (left), number of detected photons (middle) and normalized amplitude (right). The distributions of the four channels are offset in range by small amounts to align the leading edges (at the normalized amplitude $50^{\text {th }} \%$ ) in order to emphasize pulse width differences. SIMPL channels: light green $=$ green perpendicular, dark green $=$ green parallel, orange $=$ NIR perpendicular, red $=$ NIR parallel

expected. NIR light that penetrates into the water is fully absorbed so the only NIR signal is $\|$ energy reflected from the specular surface, yielding a pulse width slightly broader than the instrument impulse response likely due to wind roughening of the water. The green $\|$ signal is also dominated by the specular return from the surface and therefore has a pulse width equal to that of the NIR. The amplitude and width of these returns are a function of the wind-induced surface roughness that defines the frequency distribution laser pulse incidence angles with respect to the water surface [8]. A weak green ___ signal is observed with an asymptotic tail to longer ranges due to visible light penetration and multiple-scattering within the water column.

Reflected energy from water covered by thin, smooth, specular skim ice enables fuller assessment of green penetration and scattering in the water column (Figure 2, bottom). Because the laser pulse is intercepting the smooth, specular ice at a small off-nadir incidence angle the large majority of the reflected light is directed away from the receiver causing the very weak NIR \|| return. Similarly, the green $\|$ surface return must also be very weak so its observed signal is inferred to be due to volume scattering in the water column. This is consistent with the similarity of the green $\|$ and $\_$signal amplitudes and asymptotic tails. The green $\|$ and $\_$distributions are therefore interpreted to be extinction profiles and the depth of penetration is related to the water column optical depth. Depending on the method by which the green range is determined from the water column extinction profile, the range bias with respect to the water surface could be 10 's of $\mathrm{cm}$ for this case. A range bias of that magnitude into water relative to adjacent ice would introduce meter-level errors in sea ice thickness. Note also that the peak green || amplitude is 10x weaker than that of the wind-roughened open water case so that the range precision will be substantially reduced. Skim ice on open water leads can be a common occurrence in polar region sea ice. Its presence could introduce range biases and reduced precision by the mechanism observed in the SIMPL data, thereby causing errors in ICESat-2 measurements of sea ice freeboard and derived ice thickness.

Several methods (centroid, median, mode, leading edge and Gaussian fit) for determining the range from broadened ice and water column received pulse distributions are being investigated as a function of signal-to-noise ratio (Figure 3 ). In this example, NIR and green $\|$ range distributions were assessed using data for a block of clear sea ice acquired using a laboratory breadboard version of SIMPL. For this sample the asymptotic tail of the green volume scattering distribution extends 6 nsec (0.9) beyond that of the NIR. Green range biases, relative to the NIR, and precisions were computed as a function of signal level, from 30 to 1,000 counts sampled from the green range distribution, and as a 

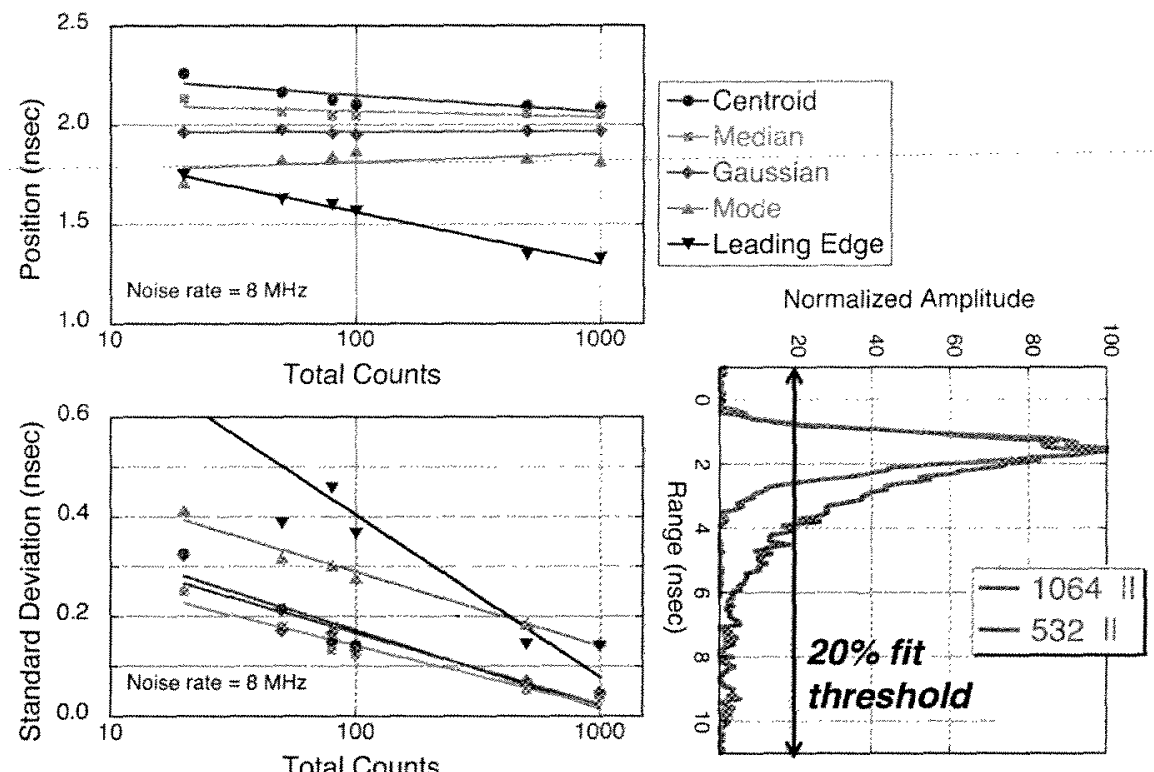

Figure 3. Assessment of range bias (top left) and precision (bottom left) as a function of signal level for 5 range determination methods, using single photon range distributions for parallel NIR and green received energy (bottom right). The position value, the measure of the range bias between green and NIR, is too large by $1.4 \mathrm{nsec}$ due to the method by which the two distributions were referenced. The sample, measured with a laboratory breadboard version of SIMPL, is a block or clear arctic sea ice provided courtesy of T. Markus. For this

example, an $8 \mathrm{MHz}$ noise rate was applied and a signal threshold equal to $20 \%$ of the peak was used for the Gaussian fit method.

function of noise rate. This example has an applied noise rate of $8 \mathrm{MHz}$. In order to reduce the effect of the tail on the Gaussian fit range bias only the signal above a threshold was used, defined as $20 \%$ of the peak signal $(10 \%$ and $30 \%$ thresholds have also been tested).

Other than the leading edge method, the range biases are relatively insensitive to signal level but they differ between the methods. The leading edge is least biased and the centroid is most biased. As expected, the range precision decreases with increasing signal level for all methods. The leading edge method has the worst precision and the median is the best. This kind of assessment, applied to airborne SIMPL range distributions for a variety of Lake Erie surface conditions, will help guide selection of an optimal range determination method for ICESat-2.

\section{CONCLUSIONS}

SIMPL's polarimetric, high-precision single-photon ranging at green and NIR wavelengths provides a unique means to assess laser pulse interactions with natural targets. Analysis of ice covered Lake Erie data is documenting and quantifying pulse-broadening and signal amplitude as a function of ice and water conditions. Resulting effects on $532 \mathrm{~nm}$ range bias and precision are being assessed by evaluating several methods for range determination. These results will aid in developing data processing and analysis methods for ICESat-2 measurements of sea ice freeboard and derived ice thickness.

\section{REFERENCES}

[1] H.J. Zwally et al., "ICESat's laser measurements of polar ice, atmosphere, ocean, and land," $J$. Geodyn., vol. 34(3-4), pp. 405-445, 2002.

[2] B.E. Schutz, H.J. Zwally, C.A. Shuman, D. Hancock, J.P. DiMarzio, "Overview of the ICESat Mission," Geophys. Res. Lett, vol. 32: L21S01, doi:10.1029/2005GL024009, 2005.

[3] R. Kwok, H.J. Zwally and D. Yi, "ICESat observations of Arctic sea ice: A first look," Geophys. Res. Lett., vol. 31:L16401, doi: 10.1029/2004GL020309, 2004.

[4] R. Kwok, G. F. Cunningham, M. Wensnahan, I. Rigor, H. J. Zwally, and D. Yi, "Thinning and volume loss of the Arctic Ocean sea ice cover: 2003-2008," J. Geophys. Res., vol. 114: C07005, doi: 10.1029/2009JC005312, 2009.

[5] W. Abdalati et al., "The ICESat-2 Laser Altimetry Mission," Proc. IEEE, vol. 98(5), pp. 735-751, 2010.

[6] D.J. Harding, "Pulsed Laser Altimeter Ranging Techniques and Implications for Terrain Mapping", in Topographic Laser Ranging and Scanning: Principles and Processing, Jie Shan and Charles Toth, eds., CRC Press, Taylor \& Francis Group, pp. 173-194, 2009.

[7] P. Dabney, D. Harding, J. Abshire, T. Huss, G. Jodor, R. Machan, J. Marzouk, K. Rush, A. Seas, C. Shuman, Xiaoli Sun, S. Valett, A. Vasilyev, A. Yu and Y. Zheng, "The Slope Imaging Multi-Polarization Photon-Counting Lidar: an Advanced Technology Airborne Laser Altimeter", Proc. Int. Geosci. Rem. Sens. Symp., 11686732, DOI 10.1109/1GARSS.2010.5650862, pp. 253-256, 2010.

[8] J.L. Bufton, F.E. Hoge and R.N. Swift, "Airborne measurements of laser backscatter from the ocean surface," Appl. Opt., vol. 22, pp. 2603-2618, 1983. 